\title{
Horner's syndrome with ipsilateral vocal cord and phrenic nerve palsies
}

\author{
RAJNI AMIN \\ M.R.C.P., F.R.C.R. \\ Radiotherapy Department, Royal Devon and Exeter Hospital, Exeter
}

\begin{abstract}
Summary
Two further cases of a recently described syndrome (Rowland Payne, 1981) constituting a triad of sympathetic ophthalmoplegia, phrenic nerve palsy and ipsilateral vagus or recurrent laryngeal nerve paralysis are described. This is a syndrome of signs, but hoarseness, dysphagia, dysaesthesia and pain in the ipsilateral shoulder may be associated symptoms. Development of this syndrome in patients with malignant disease does not necessarily signify a very grave prognosis.
\end{abstract}

KEY WORDS: breast cancer, lung cancer.

\section{Introduction}

Recently Rowland Payne (1981) reported three patients with metastatic carcinoma of the breast, who exhibited during the course of their illnesses a previously undescribed syndrome resulting from disruption of the sympathetic chain, the phrenic nerve and the innervation of the ipsilateral vocal cord. Two further cases are reported here, in a young female with carcinoma of the breast and in another female with carcinoma of the lung.

\section{Case 1}

A 41-year-old, short, obese woman presented in December 1971 with a $T_{3} N_{0} M_{0}$ carcinoma of the right breast. Following simple mastectomy she received radiotherapy to the operation scar, the right axilla and the internal mammary chain of lymph nodes. The right supraclavicular fossa was not irradiated.

She remained well until February 1976 when she developed hoarseness. Direct laryngoscopy showed paralysis of the right vocal cord. In September 1977, she also complained of dysphagia. A chest X-ray, apart from a right-sided cervical rib showed no abnormality, and a barium swallow was normal too.

In June 1978, she was noted to have a right-sided Horner's syndrome characterized by ptosis, miosis and enophthalmos. X-ray of the thoracic inlet, apart from the supernumerary rib, was normal. A chest $X$ - ray showed a raised hemidiaphragm. Diaphragmatig screening confirmed paralysis. In January 1979, she began to experience considerable pain in her righis upper limb, together with tingling and numbness along the outer aspect of her forearm and the right hand, but no neurological deficit was observed. Electromyographic studies were normal with goof sensory evoked responses. X-rays of the cervica spine were normal and a skeletal isotope scan showed. no abnormality indicative of metastases. A venogramp showed slight narrowing of the first part of the subclavian vein at the crossing of the cervical rib.

Dysphonia, dysphagia and dysaesthesia in her right forearm and hand continued to trouble her ugitib May 1979 when she lost the use of her right upheso limb. Exhaustive investigations yet again failedêtio reveal any underlying pathology. Her symptơno persisted with the ensuing disabilities for a furthe period of 12 months when she began to experience difficulty in walking as a result of weakness and lose of positional sense in her right leg. Clinically, she hae developed a partial Brown Sequard syndrome. $X \overrightarrow{0}$ rays of the entire spine were normal. A myelograns was contemplated but her health declined and she failed to keep her hospital appointment. She died in July, 1980, a few days after developing a quadripares sis. The immediate cause of her death was bronchop? neumonia. Unfortunately no post-mortem was un dertaken.

\section{Case 2}

A 65-year-old woman presented in March 198 with a 4-week history of cough, shortness of breatly. and haemoptysis. Physical examination revealed mass of nodes in her right supraclavicular fossa and impaired air entry in the right lower lobe of the lunge A chest X-ray showed a right-sided pleural effusion and right hilar enlargement with consolidation ant deflation of the right upper lobe. At bronchoscopse the main and the intermediate bronchi appeared te be the seat of the tumour. Histological examination showed squamous cell carcinoma. 


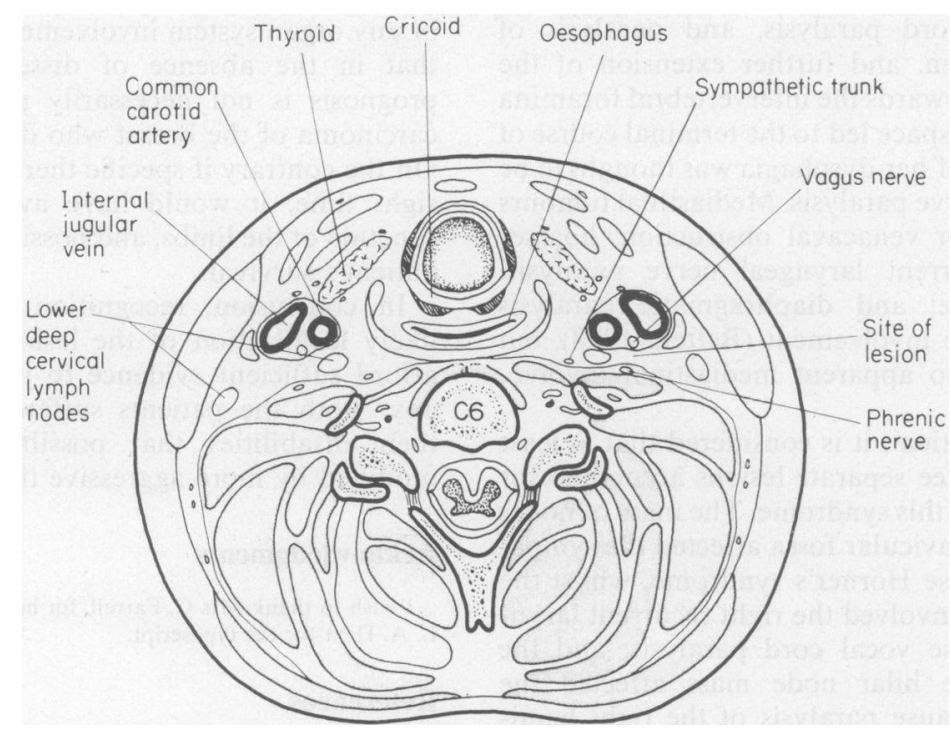

FIG. 1. Diagrammatic horizontal section of the neck at the level of cervical 6 vertebra, showing the site of the lesion in case 1

Radiotherapy eased her symptoms till June when she developed pain across the middle of her back. A chest X-ray in August showed widespread pulmonary shadowing on the right with considerably elevated right hemi-diaphragm and collapse of the right upper lobe. Diaphragmatic screening confirmed paralysis. In October 1981, she was noted to have a right-sided Horner's syndrome. A month later she developed hoarseness which seemed to disconcert her most, despite other disabling symptoms. An indirect laryngoscopy showed complete paralysis of the right vocal cord. The slow decline in her general condition continued until her death in December 1981.

\section{Discussion}

These two patients had all the features of a syndrome recently described by Rowland Payne (1981).

It is interesting to note that Hare, as long ago as 1838, in a letter to the Medical Gazette described the case of Thomas Willets, aged 40 years, 'of an unhealthy complexion', who had been admitted with a month's history of pain, tingling and and numbness along the course of the left ulnar nerve. A small tumour was palpable in the inferior triangular space of the left side of the patient's neck, his left pupil was contracted and the levator palpebrae ceased to perform its office'. The tumour became larger; the patient lost the use of his lower limbs, developed retention of urine and died soon afterwards. In a meticulous report of the post-mortem findings, Hare (1838) described hard tumour extending upward to the origin of the brachial plexus, with the carotid artery and the cervical sympathetic, vagus and phrenic nerves lost in the growth. The tumour involved the brachial plexus especially its third and fourth nerves, was attached to the spine and entered the intervertebral foramina as far as the dura with the eighth cervical and first dorsal nerves.

The first patient had no clinical, biochemical or radiological evidence of metastatic disease, and it was only towards the end that metastases to the lower deep cervical group of lymph nodes were considered as a possible cause of her signs and symptoms. Presence of a cervical rib on the affected side not only delayed the recognition of the underlying cause of her symptomatology, but also, together with the short and obese stature of the patient, it hampered accessibility to the site of the presumed metastases. The lesion accounting for the development of this syndrome in this patient was thought to be at the level of cervical 6 vertebra (Fig. 1). It is at this level that the trunks of the sympathetic, the phrenic and the vagus are in close approximation, to be affected by a single lesion. Medial to the phrenic nerve is the sympathetic trunk in the prevertebral fascia, and anteromedial are the contents of the carotid sheath (carotid artery, internal jugular vein and the vagus nerve). Metastatic infiltration of the lower deep transverse group of the internal jugular chain of lymph nodes, and involvement of the three nerve trunks accounted for the development of Horner's 
syndrome vocal cord paralysis, and paralysis of the hemi-diaphragm, and further extension of the tumour inwardly towards the intervertebral foramina and the extradural space led to the terminal course of events. The cause of her dysphagia was thought to be related to vagus nerve paralysis. Mediastinal tumours may cause superior venacaval obstruction, hoarseness due to recurrent laryngeal nerve paralysis, Horner's syndrome; and diaphragmatic paralysis from phrenic nerve involvement (Batten, 1978), but this patient had no apparent mediastinal involvement.

In the second patient, it is considered that at least two or possibly three separate lesions accounted for the development of this syndrome. The mass of nodes in the right supraclavicular fossa affected the sympathetic chain to cause Horner's syndrome, whilst the mediastinal nodes involved the right recurrent laryngeal nerve to cause vocal cord paralysis; and the mediastinal or the hilar node mass affected the phrenic nerve to cause paralysis of the right hemidiaphragm.

Development of the full syndrome in the first patient took 28 months and a further 25 months elapsed after the development of this syndrome before she died. Rowland Payne (1981) concludes that in patients with carcinoma of the breast, this is a preterminal syndrome, with a very grave prognosis.

Unlike the three previously described cases, this patient had no evidence of disseminated metastases or any organ system involvement; so it would appeaz that in the absence of disseminated spread, th\& prognosis is not necessarily poor in patients witkcarcinoma of the breast who develop this syndrome On the contrary if specific therapy was offered at the right time, it would have averted further loss of function of the limbs, and possibly even extended thi patient's survival.

In conclusion, recognition of the very discrete likely localization of the lesion as in case 1 might afford sufficient evidence to justify local radiother:apy. Both the patients suffered considerably from their disabilities that possibly could have been modified by more aggressive therapy.

\section{Acknowledgments}

I wish to thank Mrs C. Farrell, for her help with Fig. 1, and Miss L. A. Hext for the typescript.

\section{References}

Batten, J. (1978) Mediastinal tumours. In ; Price's Textbook of the Practice of Medicine (Ed. R. Bodley Scott), 12th edn., p. 927. Oxford University Press, Oxford.

HARE, E.S. (1838) Tumour involving certain nerves. Medica $\$$ Gazette, 23, 16.

Rowland PAYNe, C.M. (1981) Newly recognised syndrome in the neck: Horner's syndrome with ipsilateral vocal cord and phrenic nerve palsies. Journal of the Royal Society of Medicine, 74, 8q.

(Accepted 3 March 1983) 